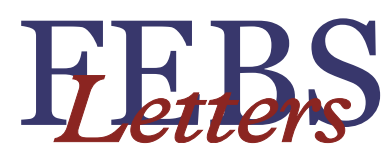

journal homepage: www.FEBSLetters.org

\title{
Regulation of HOXA2 gene expression by the ATP-dependent chromatin remodeling enzyme CHD8
}

\author{
Joel A. Yates, Tushar Menon ${ }^{1}$, Brandi A. Thompson ${ }^{1,2}$, Daniel A. Bochar * \\ The Department of Biological Chemistry, University of Michigan, Ann Arbor, MI 48109, USA
}

\section{A R T I C L E I N F O}

\section{Article history:}

Received 12 November 2009

Revised 23 December 2009

Accepted 7 January 2010

Available online 17 January 2010

Edited by Ivan Sadowski

\section{Keywords:}

Chromodomain, helicase, DNA-binding

protein 8 (CHD8)

HOX gene regulation

ATP-dependent chromatin remodeling WDR5

\begin{abstract}
A B S T R A C T
Chromodomain, helicase, DNA-binding protein 8 (CHD8) is an ATP-dependent chromatin remodeling enzyme that has been demonstrated to exist within a large protein complex which includes WDR5, Ash2L, and RbBP5, members of the Mixed Lineage Leukemia (MLL) histone modifying complexes. Here we show that CHD8 relocalizes to the promoter of the MLL regulated gene HOXA2 upon gene activation. Depletion of CHD8 enhances HOXA2 expression under activating conditions. Furthermore, depletion of CHD8 results in a loss of the WDR5/Ash2L/RbBP5 subcomplex, and consequently H3K4 trimethylation, at the HOXA2 promoter. These studies suggest that CHD8 alters HOXA2 gene expression and regulates the recruitment of chromatin modifying enzymes.

Structured summary:

MINT-7542810: CHD8 (uniprotkb:Q9HCK8) physically interacts (MI:0915) with RbBP5 (uniprotkb:Q15291) by anti tag coimmunoprecipitation (MI:0007)

MINT-7542794: CHD8 (uniprotkb:Q9HCK8) physically interacts (MI:0915) with WDR5 (uniprotkb:P61964) by anti tag coimmunoprecipitation (MI:0007)

MINT-7542820: CHD8 (uniprotkb:Q9HCK8) physically interacts (MI:0915) with ASH2L (uniprotkb:Q9UBL3) by anti tag coimmunoprecipitation (MI:0007)

MINT-7542769: CHD8 (uniprotkb:Q9HCK8) physically interacts (MI:0914) with RbBP5 (uniprotkb:Q15291), ASH2L (uniprotkb:Q9UBL3) and WDR5 (uniprotkb:P61964) by anti tag coimmunoprecipitation (MI:0007)
\end{abstract}

๑) 2010 Federation of European Biochemical Societies. Published by Elsevier B.V. All rights reserved.

\section{Introduction}

Cellular control of access to DNA is an important step in several processes including replication, repair, and transcription. Due to the chromatinized nature of eukaryotic DNA, several mechanisms of regulating the chromatin structure have evolved. These include DNA methylation, covalent modification of histones, and the use of ATP-dependent chromatin remodeling enzymes. These ATP-depen-

\footnotetext{
Abbreviations: ATRA, all-trans retinoic acid; CHD, chromodomain, helicase, DNA binding; ChIP, chromatin immunoprecipitation; DMEM, Dulbecco's Modified Eagle Medium; MLL, Mixed Lineage Leukemia; NT2/D1, NTERA2cl. D1; qPCR, quantitative PCR; RT, reverse transcription; shRNA, short hairpin RNA; WAR, WDR5/Ash2L/ RbBP5

* Corresponding author. Address: The University of Michigan Medical School, 3301E MSRB III, 1150W. Medical Center Drive, Ann Arbor, MI 48109, USA. Fax: +1 7347637799.

E-mail address: dbochar@umich.edu (D.A. Bochar).

1 Equal contribution.

2 Present address: Laboratory of Cellular and Developmental Biology, NIDDK, National Institutes of Health, Bethesda, MD 20892, USA.
}

dent remodeling enzymes use the energy from the hydrolysis of ATP to cause local changes in nucleosome position or structure.

Several families of these ATP-dependent chromatin remodeling enzymes have been defined based upon domain architecture, including the SWI/SNF, ISWI, and chromodomain, helicase, DNA binding (CHD) families. Specific to the focus of this study, the CHD family consists of nine enzymes, CHD1-9, which share in common a set of tandem chromodomains and a catalytic Snf2 helicase domain. Further classification of the CHD enzymes reveals three distinct subfamilies: CHD1-2, CHD3-5, and CHD6-9.

The Hox genes encode transcription factors that control cellular differentiation and development [1]. Hox expression is controlled by the opposing actions of the Polycomb group of repressors and the Trithorax/Mixed Lineage Leukemia (MLL) group of activators [2]. Also, the misregulation of Hox genes resulting from translocations of the MLL1 gene plays a key role in acute myeloid leukemia [3]. MLL1 is a histone H3 lysine 4 (H3K4) methyltransferase and is a member of the MLL/hSet1 family of enzymes. Common to this family is a "core" complex of WDR5, Ash2L, and RbBP5 (WAR) [4-9]. 
Here we report that CHD8 can regulate gene expression at the HOX locus. CHD8 is able to form a complex with WDR5, Ash2L, and RbBP5. CHD8 is recruited to HOX promoters and displays a significant relocalization upon gene activation using all-trans retinoic acid (ATRA). In addition, depletion of CHD8 results in a significant increase of HOXA2 gene expression upon ATRA treatment. Depletion of CHD8 also results in the loss of Ash2L at the HOXA2 promoter, suggestive of a role for CHD8 in the recruitment of the WAR complex to the HOXA2 promoter. Taken together, we demonstrate that CHD8 represses ATRA mediated HOXA2 gene expression.

\section{Materials and methods}

\subsection{Cell culture and reagents}

Dulbecco's Modified Eagle Medium (DMEM) (Invitrogen) with $10 \%$ fetal bovine serum (Hyclone) and $1 \mathrm{X}$ penicillin-streptomycin-glutamine (Invitrogen) was used to culture NTERA2cl. D1 (NT2/D1) cells at $37{ }^{\circ} \mathrm{C}$ in $10 \% \mathrm{CO}_{2}$. For ATRA induction, cells were treated with $10 \mu \mathrm{M}$ ATRA while control cells were left untreated. Cells were grown an additional $24 \mathrm{~h}$ before harvesting.

CHD8 antibodies were previously described [10]. The anti-Flag M2 (F3165) antibody and rabbit normal IgG immunoglobulin (I8140) were purchased from Sigma. The anti-RbBP5 (A300109A) and anti-Ash2L (A300-489A) antibodies were purchased from Bethyl. The anti-WDR5 (22512-100) antibody was purchased from Abcam. The anti-histone $\mathrm{H} 3$ (39163) and the anti-trimethyl histone H3 lysine 4 (39159) antibodies were purchased from Active Motif. All oligonucleotides were synthesized by Integrated DNA Technologies (Coralville, IA).

\subsection{Chromatin immunoprecipitation (ChIP) assay}

ChIP experiments were performed essentially as described in the ChIP Assay Kit (Millipore) as described previously [10].

\section{3. $R T-P C R$ and quantitative $P C R(q P C R)$}

Total RNA was isolated using the RNeasy Kit (Qiagen). Reverse transcriptase reactions employed random decamers (Ambion) and Superscript II (Invitrogen) following manufacturers' protocols. qPCR analysis employed the indicated primers (Table S1), the iQ SYBR Green Supermix or iQ Supermix (Bio-Rad), and a MyiQ Real-Time PCR Detection System (Bio-Rad). All PCR reactions were performed in triplicate. For RNA analysis, $C_{t}$ values were normalized to the levels of the Pol III transcribed H1 RNA while ChIP experiments were expressed relative to input.

\subsection{RNAi knockdown experiments}

RNAi experiments utilized the UI2-SIBR short hairpin RNA (shRNA) vectors [11] modified to express the alpha subunit of the interleukin 2 receptor as a selection marker (Table S1). CHD8 and control shRNA cassettes were described [10]. Ten micrograms were transfected into cells using lipofectamine 2000 (Invitrogen) as described by the manufacturer. Twenty four hours post-transfection, cells were either treated with ATRA or not and grown an additional $24 \mathrm{~h}$ before harvesting. Cells were trypsinized and resuspended in DMEM. Twenty microliters of Dynabeads-CD25 slurry (Invitrogen) were added and rotated at $4{ }^{\circ} \mathrm{C}$ for $30 \mathrm{~min}$. Following two PBS washes, the cells were lysed on the beads for RNA extraction or Western blotting, or, for ChIP experiments, crosslinked on the beads in $1 \%$ formaldehyde for $10 \mathrm{~min}$.

\section{Results}

\subsection{CHD8 forms a complex with the core WDR5/Ash2L/RbBP5 complex}

Previous work from our lab has identified several CHD8 associated polypeptides [10]. We reported that CHD8 can interact with WDR5 both in vivo and in vitro. Initially we focused in on WDR5 as this polypeptide has been reported to be part of a MLL histone methyltransferase complex [4-9], possibly linking chromatin remodeling and chromatin modification in a single complex. Further evidence for this link was provided by the discovery of CHD8 in a WDR5-containing complex [9]. As WDR5, Ash2L, and RbBP5 (WAR) form a subcomplex in the absence of MLL [12,13], we asked whether CHD8 could also associate with WDR5 in the context of the subcomplex. This association was tested through the reconstitution of the complex using a baculovirus expression system. Co-infection of SF9 cells with WDR5, Ash2L, RbBP5, and CHD8 followed by affinity purification demonstrated that indeed CHD8 can associate with WDR5 in the context of the WAR subcomplex (Fig. 1A).

To systematically test the assembly of the WAR/CHD8 complex, further baculovirus co-infection experiments were performed, each time omitting one component. As shown in Fig. 1A, the removal of any one of the subunits does not preclude a stable association of the remaining subunits, although omission of Ash2L results in a diminished quantity of associated RbBP5. To further explore these interactions, pairwise baculovirus co-infection experiments were performed. As shown in Fig. 1B, CHD8 is capable of interacting directly with each component of the WAR complex. These results demonstrate that CHD8 has extensive contacts with the WAR subcomplex, unlike the interactions with MLL that are dependent on WDR5 [12-15]. The precise means by which these interactions occur, including interacting domains between each protein, remains to be elucidated.

\subsection{CHD8 is bound to the promoters of the HoxA genes}

Previous studies have demonstrated that WAR is recruited to the HOX locus and is an important regulator of HOX gene expression $[8,9,12,13]$. As CHD8 has been shown to interact with WAR $[9,10]$, we investigated the possibility that CHD8 is also recruited to the HOX locus where it could function in conjunction with WAR in the regulation of transcription. The NT2/D1 embryonal carcinoma cell line was chosen as treatment of these cells with ATRA induces the expression of many genes, including the HOX genes [16], and ultimately induces cellular differentiation into neuronal-like lineages [17]. Treatment of NT2/D1 cells with ATRA results in over 500 fold activation of HOXA1, and $\sim 75-, \sim 10-$, and $\sim 4$-fold activations of HOXA2-A4, respectively (Fig. 2A). This system therefore presents an opportunity to study the role of CHD8 in Hox gene activation.

To test whether CHD8 is bound to these promoters, we first examined CHD8 occupancy in uninduced cells using ChIP assays. We found CHD8 bound to the HOXA1 and HOXA4 promoters, and to a lesser extent, the HOXA2 and HOXA3 promoters (Fig. 2B). Due to the upregulation of these HoxA genes upon treatment with ATRA, it is reasonable to suspect that proteins involved in the regulation of these genes would associate with these promoters in a dynamic manner. Therefore, we compared the occupancy of CHD8 at the HOXA1 through HOXA4 promoters before and after ATRA treatment. As shown in Fig. 2C, 24 h treatment with ATRA causes a striking relocalization of CHD8 at the HOXA1 through HOXA4 promoters, with the greatest change being the $\sim 20$-fold increase of CHD8 at the HOXA2 promoter. This result suggests that CHD8 is indeed functioning in gene regulation at the HoxA locus. 

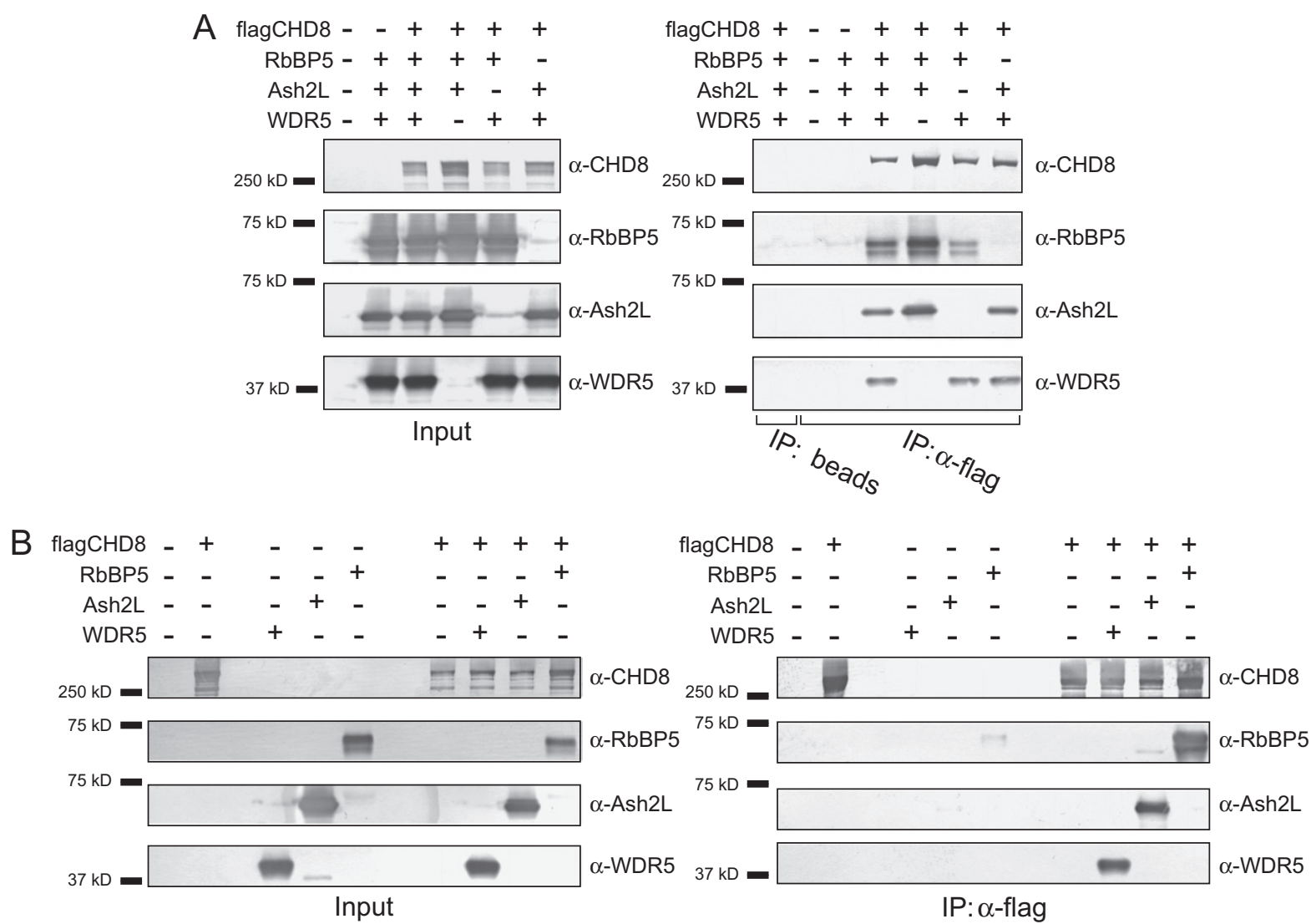

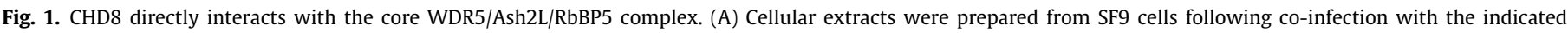

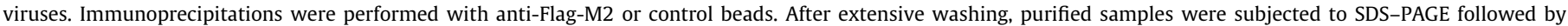
Western blotting analysis using the indicated antibodies. (B) Pairwise coinfections were performed as in (A) using the indicated baculoviruses.

\subsection{CHD8 regulates HoxA gene expression}

To directly test the effect of CHD8 upon the regulation of the HoxA locus, an shRNA strategy was employed to deplete endogenous CHD8. NT2/D1 cells were transfected with control shRNA constructs or shRNA constructs targeting CHD8. Following selection of transfected cells, cDNA was isolated for analysis by qPCR. Using this selection protocol, we were unable to detect measureable levels of HoxA gene expression in the absence of ATRA; therefore, all experiments were performed with ATRA treatment.

Depletion of endogenous CHD8 results in an increased expression of HOXA2, while the expression of HOXA1, 3, and 4 does not change in a statistically significant manner (Fig. 3A). The efficacy of CHD8 knockdown is demonstrated via Western blot in Fig. 3B. It is important to compare the increase of HOXA2 transcription in Fig. 3A to $2 \mathrm{C}$ which illustrates that, upon ATRA treatment, the HOXA2 promoter showed the greatest increase in CHD8 occupancy. Taken together, these data demonstrate that CHD8 indeed functions in the regulation of HOXA2 gene expression. Furthermore, under activating conditions at the HOXA2 promoter, the normal function of CHD8 appears to be the negative regulation of HOXA2 gene transcription. This negative effect of CHD8 on HOXA2 expression is consistent with our prior studies of CHD8 and $\beta$-catenin mediated transcription where depletion of CHD8 results in an increase in target gene expression under activating conditions [10].

\subsection{CHD8 is required for recruitment of the WAR complex}

We have previously shown CHD8 interacts with several proteins including the WAR complex. Because WAR is also involved in establishing methylation patterns at target genes $[8,9,12,13]$, we next asked whether CHD8 is involved in the recruitment of WAR to the promoters of target genes. We tested this hypothesis via knockdown of CHD8 and chromatin immunoprecipitation as above. In our hands, antibodies to Ash2L were the most suitable for these experiments. Because Ash2L has been demonstrated to be vital for WAR complex function, the absence of Ash2L is indicative of a lack of functional WAR at that gene. As expected, upon treatment with control shRNA, we are able to detect Ash2L at the HOXA2 promoter (Fig. 4). However, when CHD8 is depleted via shRNA treatment, Ash2L is lost from the promoter. This result is indicative of CHD8 being required for proper WAR complex recruitment and function. As WAR is a component of multiple histone methyltransferase complexes, CHD8, though the recruitment of Ash2L, could be required for proper establishment of methylation patterns at target genes.

\subsection{CHD8 affects histone H3 methylation patterns at the HOXA2 promoter}

As mentioned previously, the WAR complex is known to associate with all members of the SET1 family of histone H3 lysine 4 methyltransferases, playing an important regulatory role in gene expression, including transcription at the Hox loci [18]. In conjunction with the data demonstrating a requirement for CHD8 in Ash2L recruitment, a suggested role for CHD8 functioning in the regulation of histone methylation patterns at target genes emerges. We performed chromatin immunoprecipitation with H3K4me3 antibodies to look for changes in methylation at the HOXA2 promoter following knockdown of CHD8 and ATRA treatment. Consistent 
A

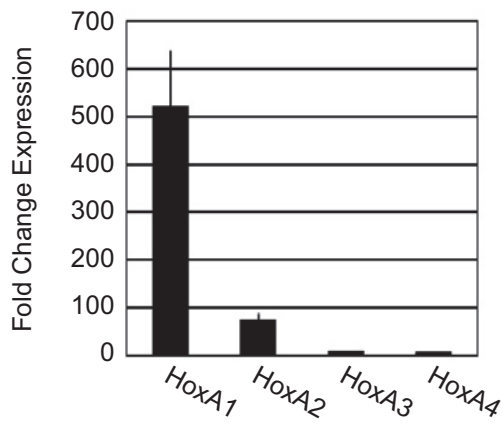

B
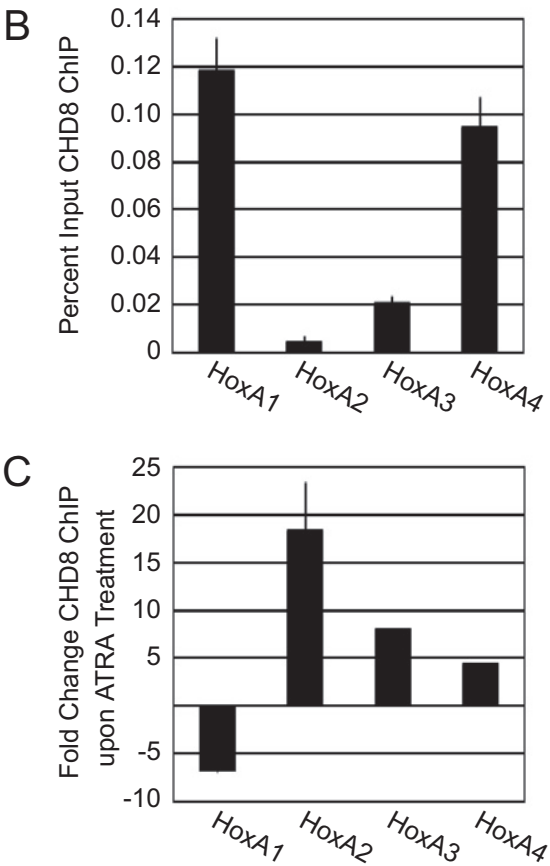

Fig. 2. CHD8 is bound to the HoxA gene cluster. (A) NT2/D1 cells were treated with ATRA for $36 \mathrm{~h}$. Cells were harvested and RNA was isolated. Expression of the indicated genes was analyzed by RT-PCR. (B) Chromatin from NT2/D1 cells was crosslinked with formaldehyde. Cells were lysed, and ChIPs were performed with anti-CHD8 antibodies. Bound DNA was detected by quantitative PCR with primer pairs to the promoter region of the indicated gene. (C) ChIP experiments were performed using NT2/D1 cells that had been treated with ATRA or not. Results are expressed relative to untreated cells. Control IgG precipitated samples in all experiments were less than $0.001 \%$ of input and not shown.

with the loss of Ash2L, we found a significant decrease in H3K4me3 levels at the HOXA2 upon depletion of CHD8 (Fig. 5A).

One possible explanation for the decrease in $\mathrm{K} 4$ trimethylation is that CHD8 functions in histone deposition at the HOXA2 promoter, i.e. the loss of observed methylation is due to loss of total $\mathrm{H} 3$ at this promoter. To address this, we again performed chromatin immunoprecipitation experiments after depletion of CHD8 by shRNA and treatment with ATRA. As shown in Fig. 5B, H3 occupancy at the HOXA2 promoter increases upon CHD8 depletion. This result suggests that the regulation of transcription by CHD8 occurs via multiple histone methylation/demethylation events at the promoters of target genes.

\section{Discussion}

Here, we demonstrate the formation of a CHD8/WDR5/Ash2L/ RbBP5 complex. Also shown is the localization of CHD8 to the HOXA2 promoter where it acts to negatively regulate expression under activating conditions. Depletion of CHD8 results in the loss

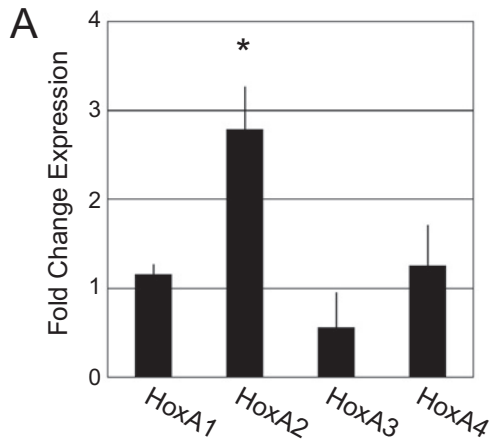

B

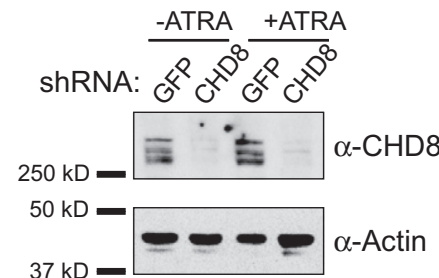

Fig. 3. CHD8 regulates expression of the HoxA gene cluster. (A) NT2/D1 cells were transfected with control shRNA or CHD8 shRNA and induced with ATRA. Following selection, cells were harvested and total RNA was isolated. Expression of the indicated genes was analyzed by RT-qPCR. Results are normalized first to a reference RNA and then to the control shRNA transfection. ${ }^{*} P<0.03$ by Student's $t$ test. (B) Western blot of control transfected shRNA (GFP) or CHD8 transfected shRNA cellular lysate. Actin was used as a loading control.
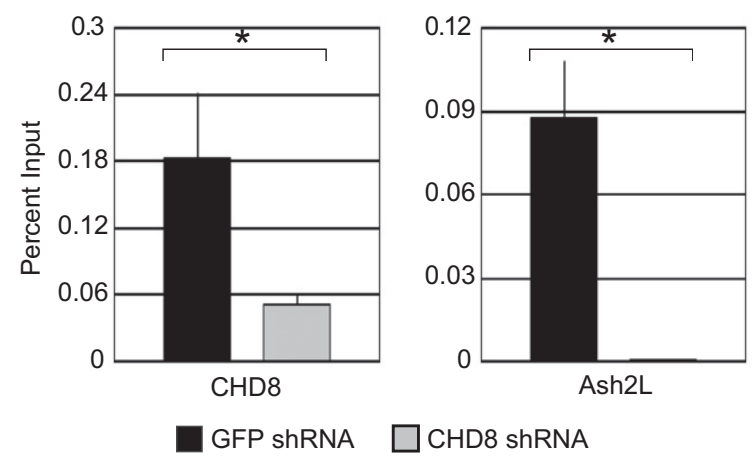

Fig. 4. CHD8 is required for the recruitment of Ash2L to the HOXA2 promoter. NT2 D1 cells were transfected with control shRNA or CHD8 shRNA and treated with ATRA. Following selection of the transfected cells, cells were cross linked using formaldehyde. Cells were then lysed, following which ChIP experiments were performed using the indicated antibodies. Bound DNA was detected by quantitative PCR with primer pairs to the promoter region of HOXA2. Control IgG precipitated samples were less than $0.001 \%$ of input and therefore are not shown. ${ }^{*} P<0.05$ by Student's $t$-test.

of Ash2L as well as loss of the H3K4me3 histone mark at the HOXA2 promoter. These data collectively demonstrate a role for CHD8 in regulation of HOXA2 gene expression.

We demonstrate a regulatory role for CHD8 at HOXA2, but the mechanistic details of CHD8 function remain to be fully elucidated. Though our data demonstrates a role for CHD8 in the maintenance of promoter environments, the data are inconsistent regarding the mode of action of CHD8 at this promoter. On one hand, the expression data demonstrates a role for CHD8 in the negative regulation of target genes (Fig. 3), while ChIP data indicates that CHD8 is necessary for the recruitment and action of a histone modifying complex known to activate transcription at target promoters (Figs. 4 and 5). Further studies into the biological consequences of CHD8 


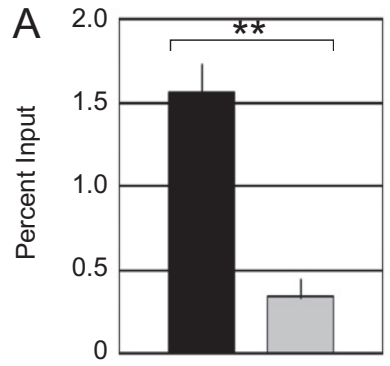

CHD8

GFP ShRNA

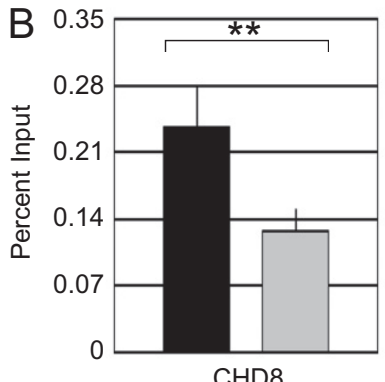

GFP shRNA

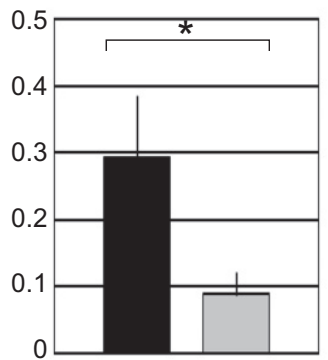

H3K4me3

CHD8 shRNA

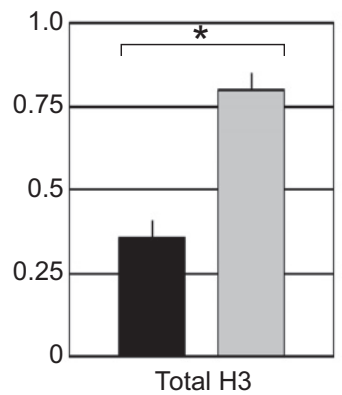

CHD8 shRNA
Fig. 5. CHD 8 affects H3K4 trimethylation at the HOXA2 promoter. (A and B) ChIP experiments were performed as in Fig. 4 using the indicated antibodies. Bound DNA was detected by quantitative PCR with primer pairs to the promoter region of HOXA2. Control IgG precipitated samples were less than $0.001 \%$ of input and therefore are not shown. ${ }^{*} P<0.05$ by Student's $t$-test and ${ }^{* *} P<0.01$ by Student's $t$ test.

loss as well as further biochemical characterization of CHD8 are required to understand the role or roles CHD8 is playing at target promoters.

CHD8 has been demonstrated to function within the cell in multiple contexts and with a variety of binding partners [19-22]. These studies, in combination with the current work demonstrate the important, though cryptic, role of CHD8 within the cell. In addition, if MLL1 and CHD8 are working to cooperatively regulate genes, the potential exists for erroneous regulation of CHD8 and leukemogenic MLL1 fusion proteins to exacerbate transcriptional misregulation in cancer. Thus, CHD8 may prove to be an effective target for novel therapeutics. Further experiments probing the mechanisms of CHD8 targeting, binding, and in vivo activity are needed to understand the cellular role of this important remodeling enzyme.

\section{Acknowledgements}

We thank R.C. Trievel, J.F. Couture, V. Tremblay, and Y. Dou for useful reagents. We thank R.P. Kwok, R.C. Trievel, M.D. Uhler for critical reading of this manuscript. This work was funded by an American Cancer Society Research Scholar Grant (RSG-07-189$01)$ to D.A.B.

\section{Appendix A. Supplementary data}

Supplementary data associated with this article can be found, in the online version, at doi:10.1016/j.febslet.2010.01.022.

\section{References}

[1] Martinez, P. and Amemiya, C.T. (2002) Genomics of the HOX gene cluster. Comp. Biochem. Physiol. B: Biochem. Mol. Biol. 133, 571-580.

[2] Ringrose, L. and Paro, R. (2004) Epigenetic regulation of cellular memory by the polycomb and trithorax group proteins. Annu. Rev. Genet. 38, 413-443.

[3] Andreeff, M. et al. (2008) HOX expression patterns identify a common signature for favorable AML. Leukemia 22, 2041-2047.

[4] Miller, T., Krogan, N.J., Dover, J., Erdjument-Bromage, H., Tempst, P., Johnston, M., Greenblatt, J.F. and Shilatifard, A. (2001) COMPASS: a complex of proteins associated with a trithorax-related SET domain protein. Proc. Natl. Acad. Sci. USA 98, 12902-12907.

[5] Wysocka, J., Myers, M.P., Laherty, C.D., Eisenman, R.N. and Herr, W. (2003) Human Sin3 deacetylase and trithorax-related Set1/Ash2 histone H3-K4 methyltransferase are tethered together selectively by the cell-proliferation factor HCF-1. Genes Dev. 17, 896-911.

[6] Goo, Y.H. et al. (2003) Activating signal cointegrator 2 belongs to a novel steady-state complex that contains a subset of trithorax group proteins. Mol. Cell Biol. 23, 140-149.

[7] Yokoyama, A., Wang, Z., Wysocka, J., Sanyal, M., Aufiero, D.J., Kitabayashi, I., Herr, W. and Cleary, M.L. (2004) Leukemia proto-oncoprotein MLL forms a SET1-like histone methyltransferase complex with menin to regulate Hox gene expression. Mol. Cell Biol. 24, 5639-5649.

[8] Wysocka, J. et al. (2005) WDR5 associates with histone H3 methylated at K4 and is essential for H3 K4 methylation and vertebrate development. Cell 121, 859-872.

[9] Dou, Y. et al. (2005) Physical association and coordinate function of the H3 K4 methyltransferase MLL1 and the H4 K16 acetyltransferase MOF. Cell 121, 873885.

[10] Thompson, B.A., Tremblay, V., Lin, G. and Bochar, D.A. (2008) CHD8 is an ATPdependent chromatin remodeling factor that regulates beta-catenin target genes. Mol. Cell Biol. 28, 3894-3904.

[11] Chung, K.H., Hart, C.C., Al-Bassam, S., Avery, A., Taylor, J., Patel, P.D., Vojtek, A.B. and Turner, D.L. (2006) Polycistronic RNA polymerase II expression vectors for RNA interference based on BIC/miR-155. Nucleic Acids Res. 34, e53.

[12] Dou, Y., Milne, T.A., Ruthenburg, A.J., Lee, S., Lee, J.W., Verdine, G.L., Allis, C.D. and Roeder, R.G. (2006) Regulation of MLL1 H3K4 methyltransferase activity by its core components. Nat. Struct. Mol. Biol. 13, 713-719.

[13] Steward, M.M., Lee, J.S., O'Donovan, A., Wyatt, M., Bernstein, B.E. and Shilatifard, A. (2006) Molecular regulation of H3K4 trimethylation by ASH2L, a shared subunit of MLL complexes. Nat. Struct. Mol. Biol. 13, 852-854.

[14] Patel, A., Vought, V.E., Dharmarajan, V. and Cosgrove, M.S. (2008) A conserved arginine-containing motif crucial for the assembly and enzymatic activity of the mixed lineage leukemia protein-1 core complex. J. Biol. Chem. 283, 32162-32175.

[15] Song, J.J. and Kingston, R.E. (2008) WDR5 interacts with mixed lineage leukemia (MLL) protein via the histone H3-binding pocket. J. Biol. Chem. 283 , 35258-35264.

[16] Mavilio, F., Simeone, A., Boncinelli, E. and Andrews, P.W. (1988) Activation of four homeobox gene clusters in human embryonal carcinoma cells induced to differentiate by retinoic acid. Differentiation 37, 73-79.

[17] Andrews, P.W. (1984) Retinoic acid induces neuronal differentiation of a cloned human embryonal carcinoma cell line in vitro. Dev. Biol. 103, 285-293.

[18] Hess, J.L. (2004) MLL: a histone methyltransferase disrupted in leukemia. Trends Mol. Med. 10, 500-507.

[19] Ishihara, K., Oshimura, M. and Nakao, M. (2006) CTCF-dependent chromatin insulator is linked to epigenetic remodeling. Mol. Cell 23, 733-742.

[20] Nishiyama, M. et al. (2009) CHD8 suppresses p53-mediated apoptosis through histone H1 recruitment during early embryogenesis. Nat. Cell Biol. 11, 172182.

[21] Rodriguez-Paredes, M., Ceballos-Chavez, M., Esteller, M., Garcia-Dominguez, M. and Reyes, J.C. (2009) The chromatin remodeling factor CHD8 interacts with elongating RNA polymerase II and controls expression of the cyclin E2 gene. Nucleic Acids Res. 37, 2449-2460.

[22] Yuan, C.C., Zhao, X., Florens, L., Swanson, S.K., Washburn, M.P. and Hernandez, N. (2007) CHD8 associates with human Staf and contributes to efficient U6 RNA polymerase III transcription. Mol. Cell Biol. 27, 8729-8738. 